\title{
Actions de sensibilisation à la microélectronique envers les lycéens dans le cadre du projet Nano@school
}

\author{
E. Excoffon ${ }^{\mathrm{a}}$, A. Bsiesy ${ }^{\mathrm{b}}$, S. Berger ${ }^{\mathrm{c}}$, O. Bonnaud $^{\mathrm{d}, \mathrm{e}}$, \\ anspection Pédagogique, Rectorat de l'Académie de Grenoble, France \\ ${ }^{\mathrm{b}}$ CIME Nanotech, Université Grenoble Alpes, Grenoble, France \\ ${ }^{\mathrm{c}}$ CEA Tech, Grenoble, France \\ 'Université de Rennes 1, France \\ ${ }^{\mathrm{e}}$ GIP-CNFM, Grenoble, France \\ Contact email : olivier.bonnaud@univ-rennes1.fr
}

Ce papier présente les expériences de sensibilisation à la microélectronique et aux nanotechnologies de lycéens menées dans le cadre du programme Nano@school à Grenoble. A l'origine de ce programme, qui fête ses dix années d'existence en 2019, se trouvent trois partenaires : le CEA de Grenoble, le pôle CNFM de Grenoble (CIME Nanotech), et le Rectorat de Grenoble. Depuis 2012, ce programme est inclus dans une des actions du projet piloté par le réseau national de formation à la microélectronique (CNFM) et dont le CIME Nanotech est un membre majeur. Il est en effet très important de pouvoir attirer les jeunes dans ces domaines scientifiques qui sont souvent mal connus et où cependant la demande de main d'œuvre qualifiée est importante. Les sessions se déroulant sur une journée proposent aux lycéens de véritables mini-projets scientifiques, à travers des expérimentations encadrées par des enseignants et/ou chercheurs sur des plateformes technologiques ayant des équipements de pointe. La sensibilisation est donc basée essentiellement sur un travail pratique dans un environnement de formation universitaire et de recherche. Le retour d'expérience indique que l'approche est globalement très bien perçue par les lycéens et par leurs professeurs, très motivante pour les organisateurs et encadrants sur les plateformes, mais son impact sur l'attractivité reste néanmoins difficile à mesurer à court terme.

\section{Introduction : nécessité d'une sensibilisation des jeunes}

L'évolution des technologies électroniques depuis plus de 50 ans a permis l'introduction dans le monde d'un grand nombre de produits manufacturés qui améliorent et qui facilitent l'information, les communications, la mobilité (transports), mais également la santé, l'environnement, l'énergie, et la sécurité pour ne citer que les plus visibles (1). Tous ces produits ont pour cœur l'électronique et la microélectronique, partie physique de la génération et du traitement de l'information essentiellement numérique (2). L'apparition relativement récente des objets connectés a accentué d'une part cette évolution et d'autre part l'importance de l'ingénieur dans cette discipline qui représente à ce jour un chiffre d'affaire mondial de 500 milliards de dollars mais qui induit dans l'ensemble des activités sociétales un chiffre d'affaire mondial bien supérieur et voisin de 40.000 milliards de dollars (3). 
Les composants et systèmes sont ainsi de plus en plus complexes, nécessitent de plus en plus de liens avec les domaines d'applications et donc de plus en plus de compétences en ingénierie de l'électronique et des systèmes en parallèle aux sciences de l'informatique, de la robotique et de l'intelligence artificielle (4). Toutefois, les sociétés actuelles par l'intermédiaire des médias mettent de moins en moins en valeur ces domaines techniques, scientifiques et technologiques, malgré le besoin croissant de ces compétences au niveau des entreprises, de la recherche et de l'innovation. Paradoxalement, les jeunes, premiers utilisateurs de ces technologies, se désintéressent de ces domaines d'activité, et plus largement de la connaissance sous-jacente. Le marché du travail est actuellement contraint par le faible nombre de candidatures de techniciens, ingénieurs et docteurs capables d'alimenter la filière électronique au niveau français mais également européen, voire international. Il est donc important de sensibiliser les jeunes, au niveau des études secondaires, à ce monde qui leur est étranger et de leur montrer l'intérêt scientifique et professionnel de ces disciplines (5).

C'est dans cet objectif qu'a été conçu le programme «Nano@school ». Il a pu être mis en place par la volonté affirmée de trois acteurs: des enseignants-chercheurs de l'enseignement supérieur, des chercheurs des centres de recherche et transfert tel que le CEA et de l'inspection pédagogique de Physique-Chimie du Rectorat d'académie de Grenoble, de bâtir une expérience de sensibilisation de lycéens, de préférence dans des classes à vocation scientifique, et de la prolonger en cas de succès (6). L'objectif final est de développer l'attractivité de ce domaine d'activité et d'augmenter sensiblement le vivier de compétences techniques et scientifiques afin d'alimenter la filière électronique française, à minima.

Cet article traite ainsi de la mise en place de la première expérimentation de sensibilisation des lycéens, et de son développement aussi bien sur le plan quantitatif que qualitatif (7), pour atteindre le niveau actuel. Des exemples d'activités pratiques offertes aux lycéens par le CIME Nanotech (8), centre interuniversitaire de microélectronique de Grenoble et pôle grenoblois du Groupement d'intérêt public le GIP-CNFM (9), sont détaillés. La dernière partie traite du retour d'expérience et de la dissémination de cette opération dans le cadre du réseau national GIP-CNFM et plus particulièrement avec le support du programme IDEFI-FINMINA (10-12).

\section{Historique et soutien du programme Nano@school}

Le programme Nano@school a démarré par des sessions expérimentales entre 2008 et 2010 avec deux classes de lycées de l'Académie de Grenoble dont les élèves sont venues sur le site du pôle microélectronique grenoblois. Ces élèves ont pu être sensibilisés sur plusieurs plateformes du CIME Nanotech. Ils ont pu mener une expérience en salle blanche microélectronique leur permettant de réaliser une opération de photolithogravure, étape clef de toutes les fabrications de composants et circuits intégrés microélectroniques. Ils ont pu être aussi sensibilisés aux techniques d'observation et de caractérisation à l'échelle nanométrique (plateforme intitulée «nanomonde») et aux logiciels de conception assistée par ordinateur leur permettant de comprendre comment concevoir des circuits intégrés comportant plusieurs milliards de composants électroniques élémentaires tels que des transistors à effet de champ (MOSFET). Fort de cette première expérience concluante (6) le programme s'est structuré sous sa forme actuelle à partir de l'année scolaire 2010-2011, avec 12 classes sélectionnées et réceptionnées sur les plateformes du pôle. Cette montée en puissance a également correspondu à une extension de l'accès aux plateformes, en introduisant au menu la plateforme «Photovoltaïque », la plateforme «Biotechnologie » et la plateforme « Microsystèmes ». 
La figure 1 montre l'historique du programme qui s'est ensuite développé et consolidé pour aboutir à la situation actuelle (13). Pour cette dernière année académique 2017-2018, 23 sessions ont été organisées, auxquelles se sont ajoutées 4 journées pour des classes de lycées internationaux (Japon, Italie et Turquie).

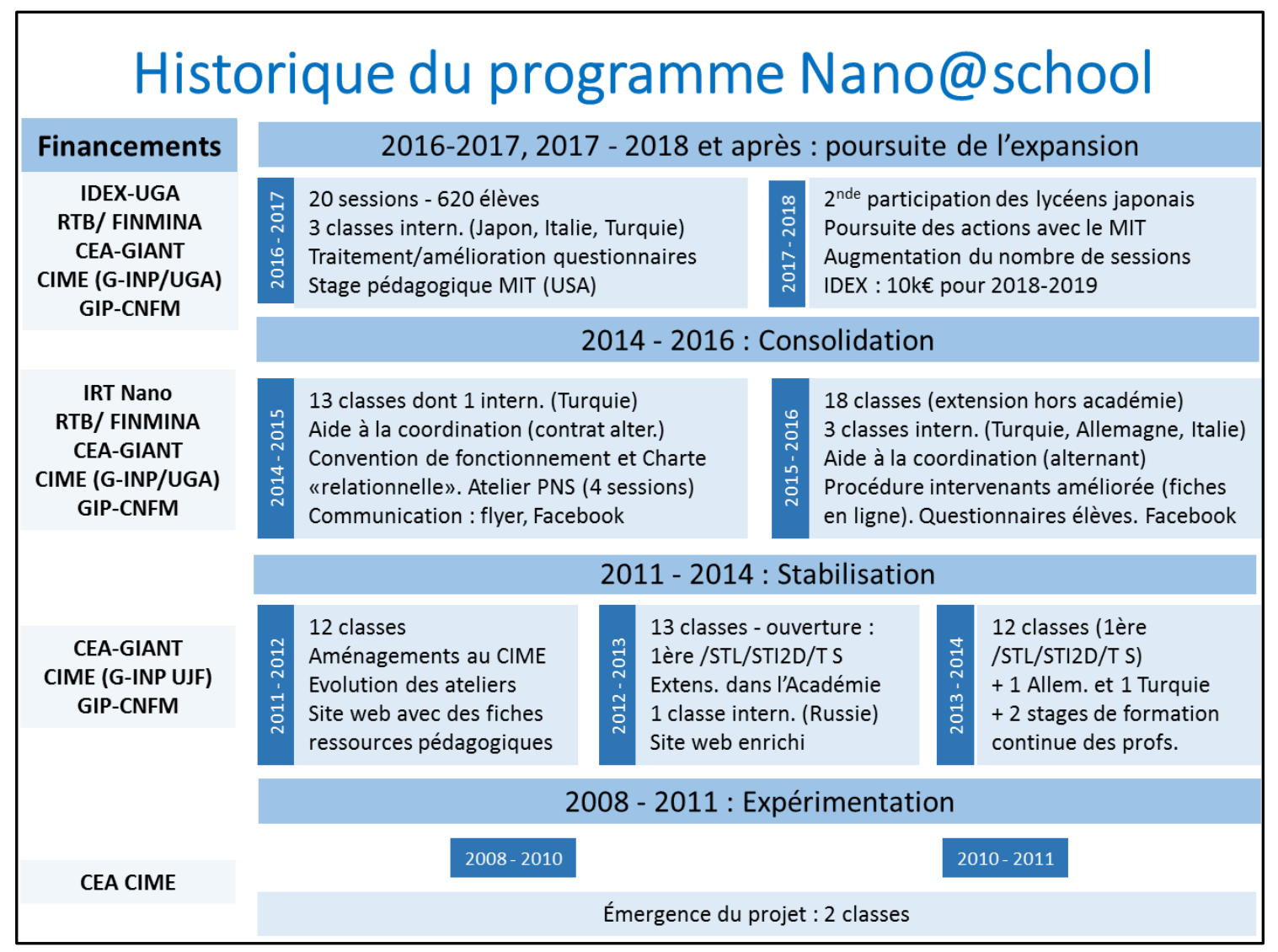

Fig. 1 Historique du programme Nano@school depuis 2008 et évolution des soutiens financiers. Après 2 années d'expérimentation de faisabilité (6), le programme s'est étoffé pour atteindre au cours des 3 dernières années académiques la sensibilisation de plus de 800 lycéens chaque année (13).

Concernant le financement, cette sensibilisation n'apparaissait pas explicitement dans les actions des pôles interuniversitaires CNFM, essentiellement dédiées à la formation des étudiants de l'enseignement supérieur jusqu'au niveau du doctorat et aux professionnels dans le cadre de la formation continue ou tout au long de la vie (14). Mais sous l'impulsion des partenaires industriels manifestant ce besoin criant, des actions volontaristes ont été structurées entre le pôle de Grenoble (CIME Nanotech) soutenu par le Conseil d'Administration du GIP-CNFM, et CEATech dans le cadre des actions GIANT, avec un intérêt affiché de l'Académie de Grenoble qui pouvait insérer ces projets technologiques originaux pour les lycéens dans leur programme pédagogique. Sans pouvoir abonder financièrement directement, grâce à l'engagement de l'Inspection Pédagogique Régionale, l'Académie de Grenoble contribue efficacement par la mise à disposition à temps partiel d'un professeur du Lycée Cité Scolaire Internationale qui apporte son soutien pédagogique au programme. Les lycées sélectionnés pour participer au programme prennent en charge le déplacement de leurs élèves, sachant qu'il n'y a pas de ségrégation sur l'ensemble de l'académie et que certaines classes ont plus de deux heures de transport pour atteindre le pôle grenoblois depuis leur ville d'origine. 
Depuis 2012, le GIP-CNFM étant coordonnateur d'un projet FINMINA (10) du programme IDEX Formation ou IDEFI inclus dans le Plan d'Investissement d'Avenir, afin de répondre à cette stratégie d'attractivité et de sensibilisation, une des 8 actions est consacrée à la sensibilisation des lycéens. Ce projet FINMINA peut ainsi contribuer financièrement à cette opération Nano@school. Avec en parallèle une implication de l'Institut de Recherche Technologique Nanoelec piloté par les établissements d'enseignement supérieur grenoblois, qui ont chacun un compartiment de soutien à la formation, et, par ailleurs, des soutiens ponctuels de 1'IDEX-UGA à la mise en place de nouveaux ateliers, ce programme est totalement financé.

\section{III.Description du programme}

Nano@school est un programme dans lequel une classe de $1^{\text {ère }}$ ou de Terminale scientifique ou technologique s'engage sur la durée, sous le contrôle de l'Inspection d'académie de Grenoble. La journée au CIME Nanotech, point culminant du projet de classe Nano@school, se place dans le cadre d'un véritable projet de classe qui se déroule sur plusieurs semaines. En amont: travail sur les concepts scientifiques qui seront abordés et sur la démarche expérimentale; en aval: partage et exploitation des expériences des différents ateliers. Une production demandée aux classes, permet la retranscription de l'expérience et des connaissances acquises. Cette dimension est fondamentale pour que la journée au CIME Nanotech, extrêmement riche, puisse être exploitée au mieux, à l'opposé d'une simple visite de laboratoire, ponctuelle et sans lendemain. La session expérimentale se déroule sur une journée au CIME Nanotech que la classe atteint après un déplacement qui peut durer jusqu'à $2 \mathrm{~h}$. Elle débute à $9 \mathrm{~h}$ du matin par une introduction au domaine de la microélectronique et une présentation des installations et des actions du pôle. Les lycéens sont ensuite répartis par groupes de 4-8 élèves dans leurs ateliers respectifs. Les ateliers se déroulent sur les différentes plateformes du CIME Nanotech, et sont encadrés par des enseignants-chercheurs ou des chercheurs, assistés des responsables technologiques des plateformes. Les lycéens suivent un atelier le matin et un autre l'après-midi, de $2 \mathrm{~h} 30$ chacun (le déjeuner leur est offert au restaurant universitaire). A la fin de la journée, vers 16h00, tous les lycéens sont regroupés pour effectuer avec les encadrants et leurs enseignants une synthèse durant laquelle ils remplissent un questionnaire. Cette dernière action permet le premier retour d'expérience sachant qu'un second retour sera effectué au niveau des lycées en fin de module dédié.

\section{IV.Sélection des lycées et formation des professeurs}

En début d'année académique, la gouvernance de Nano@school lance un appel d'offre signé du Recteur à l'ensemble des établissements d'enseignement secondaire de l'académie. La sélection des lycées se fait à la mi-octobre par l'Inspecteur Pédagogique Régional en charge du programme, en accord avec l'équipe pédagogique et technique.

Les lycées qui participent à Nano@ school proviennent essentiellement de l'Académie de Grenoble. Il faut remarquer que certains viennent de départements reculés, et font jusqu'à quatre heures de trajet aller-retour pour profiter de cet accès au CIME Nanotech. Une des particularités du programme est aussi d'accueillir des lycées Internationaux en

provenance de pays étrangers, comme l'Italie, ou l'Allemagne mais aussi le Japon ou la Turquie. Dans ces derniers cas, le passage au pôle CNFM de Grenoble s'inscrit dans un programme pédagogique et culturel se déroulant sur plusieurs jours. 
Afin d'avoir une structure pédagogique bien construite dans les lycées, les enseignants encadrant les lycéens lors de leur venue au centre suivent eux-mêmes une formation. Ainsi, chaque année, début novembre, une formation des professeurs des lycées est organisée sur une journée. Celle-ci se fait en deux temps.

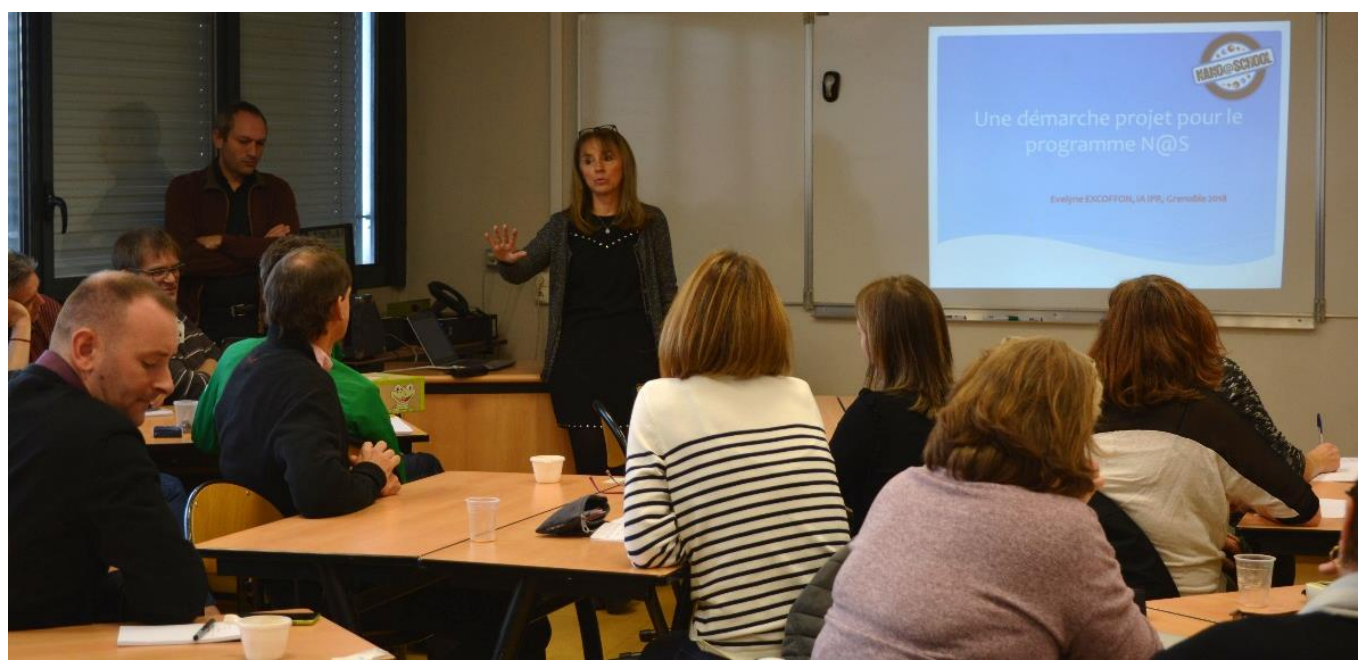

Fig. 2 Formation des professeurs en début d'année académique. La formation comprend une demijournée théorique en lycée et une demi-journée pratique sur les plateformes sélectionnées pour leurs élèves.

La matinée a lieu au Lycée Europole ( $c f$. figure 2). L'inspectrice d'Académie ouvre la session de formation de formateurs par une présentation générale du cadre institutionnel. Puis chacun des professeurs présente ses propres travaux engagés avec leurs élèves dans le cadre du module pédagogique mis en place dès la sélection des établissements lauréats. L'après-midi est consacré, au CIME Nanotech, à la familiarisation aux ateliers et aux expérimentations proposées aux lycéens. Cette opération facilite lors de leur venue l'encadrement de leurs élèves par les collègues responsables de l'accueil et du fonctionnement opérationnel sur plateformes.

\section{Ateliers proposés}

L'offre Nano@school comprend actuellement 8 ateliers clairement identifiés et correspondant à des manipulations adaptées aux lycéens qui n'ont pas le même bagage théorique que des étudiants au niveau fin de licence ou master! Ces ateliers sont les suivants :

- Nanomonde - caractérisation à l'échelle nanométrique de couches de surfaces ou de composants de très forte intégration, initiation à l'imagerie de nano-objets,

- Salle blanche technologique de fabrication de composants et circuits microélectroniques,

- Photovoltaïque - analyse et caractérisation opérationnelle de cellules photovoltaïques à base de semiconducteurs,

- Biotechnologies - mise en œuvre des techniques d'analyse de couches biologiques s'intégrant dans des capteurs appliqués à la biologie et à la médecine

- Microfluidique - analyse et caractérisation d'objets alliant les microsystèmes fluidiques (micropompes, mélangeurs) et la microélectronique associée,

- Télécom - caractérisation et analyse des techniques de transmissions et de réception de signaux hautes fréquences,

- Microsystèmes - analyse et caractérisation d'objets alliant la micromécanique et la microélectronique associée, 
- Electronique bas coût. : compréhension de la composition et du fonctionnement d'objets technologiques du quotidien (disque dur, carte FPGA, écran de téléphone portable); recyclage de ces composants pour la réalisation de petits systèmes électroniques originaux à l'aide de cartes Arduino et de capteurs, etc.... C'est la justification du bas coût !

En fonction de la disponibilité des encadrants du centre, les classes se voient proposer des ateliers pour leur journée de présence, programmée dès le premier trimestre académique. La figure 3 illustre deux ateliers suivis par des lycéens, d'une part sur la plateforme biotechnologie, et d'autre part dans la salle blanche microélectronique.
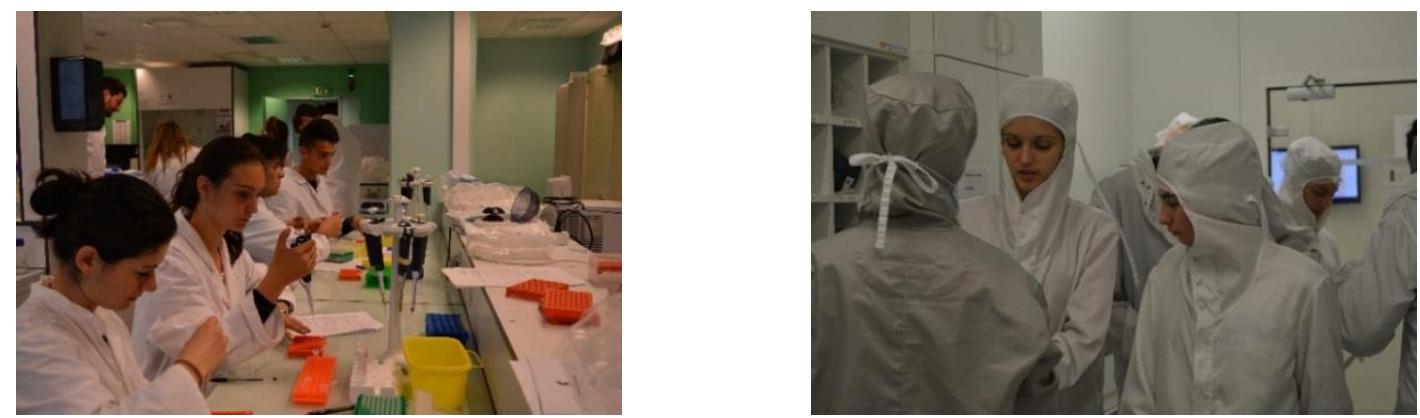

Fig. 3 Exemples d'ateliers réalisés au CIME par les lycéens. La photo de gauche présente l'atelier biotechnologies tandis que la photo de droite a été prise dans le hall de la salle blanche du CIME lors de l'habillage spécifique.

\section{Retour d'expériences}

Comme déjà évoqué, les élèves remplissent individuellement un questionnaire en ligne en fin de session sur des tablettes qui ont été acquises à cette intention, et qui sont connectées à un serveur local, ce dernier permettant d'effectuer une synthèse rapide des réponses de l'ensemble des élèves et surtout de les rentrer dans une base de données mémorisée. Les classes peuvent comporter jusqu'à 40 lycéens qui passent la journée au sein du centre et cette opération a donc été automatisée par l'équipe d'encadrement du pôle.

La figure 4 présente une page du questionnaire. Les questions concernent les ateliers mais aussi les souhaits d'orientation futurs et des questions ouvertes. Une présentation très synthétique et interactive facilite le remplissage du questionnaire qui ne nécessite que quelques minutes au maximum.

Les retours sont globalement très positifs. Certains ateliers sont particulièrement prisés comme l'atelier salle blanche dans laquelle les lycéens réalisent physiquement sur des substrats de silicium une gravure comportant des motifs micrométriques par procédé photographique en lumière ultra-violette qui est caractéristique de la fabrication des circuits intégrés. Chaque lycéen traite sa propre plaquette. La fierté sous-jacente de réussir sa propre «photolithogravure » est très stimulante et cette réalisation ouvre très fortement l'observation, la curiosité et suscite également des questions souvent très pertinentes posées aux encadrants.

Il est à noter que les filles sont majoritaires dans certaines classes, ce qui laisse espérer que la gente féminine pourra être mieux représentée dans ce domaine de l'ingénierie dans quelques années, alors que leur représentation actuelle dans ces disciplines est fortement minoritaire et voisine de $15 \%$ ! 

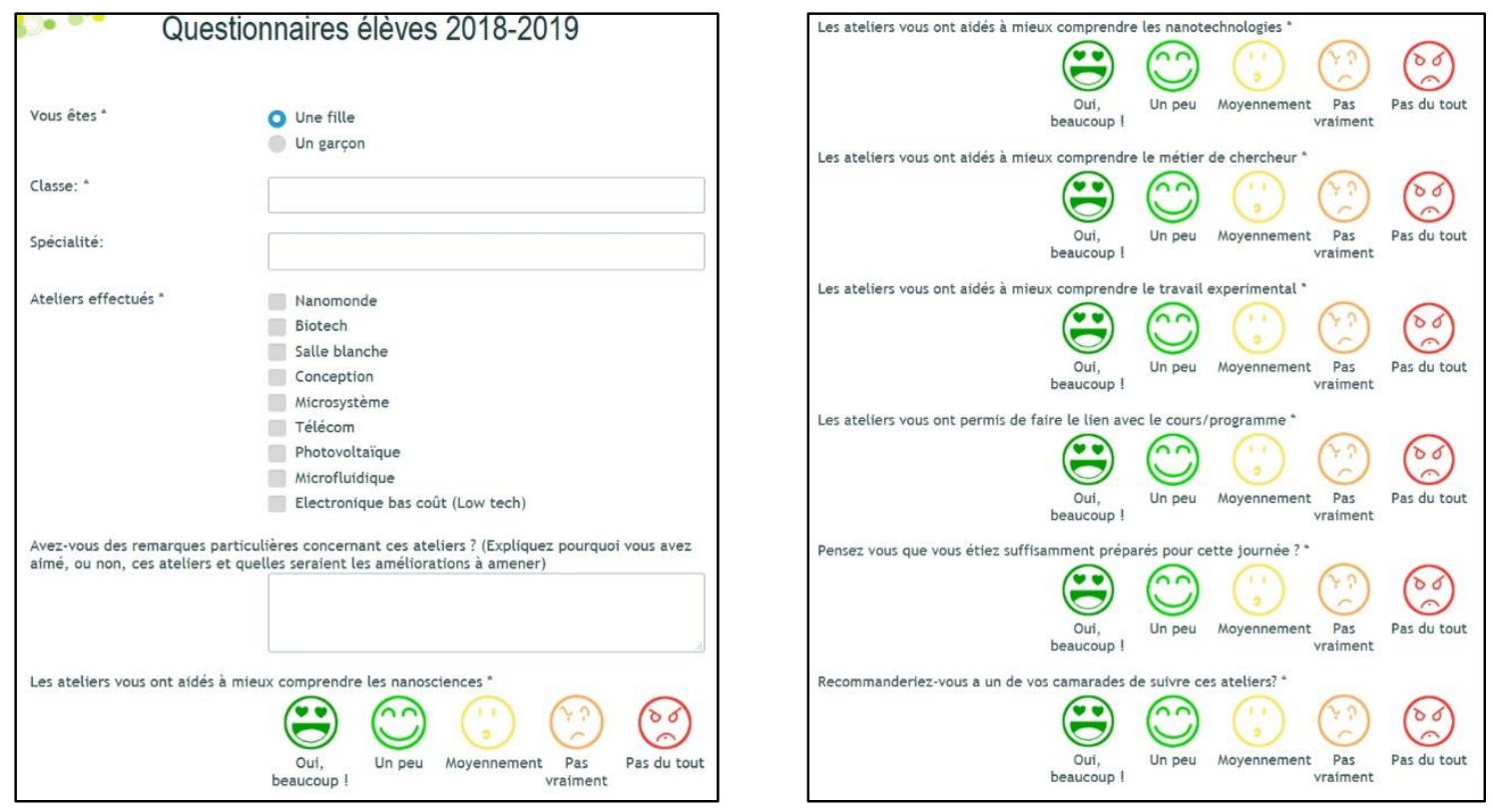

Fig. 4 Les deux premières pages du questionnaire-type rempli par les lycéens en fin de session sur le site du CIME Nanotech. Les questions concernent d'une part les ateliers, et d'autre part leur souhait d'orientation future.

A la fin des modules pédagogiques en lycée, une forte proportion du corps professoral organise dans son propre lycée des séminaires de synthèse au cours desquels les lycéens peuvent être amenés à faire des exposés devant les élèves d'autres classes qui n'ont pas pu être sélectionnées. Cela permet d'étendre la sensibilisation.

Enfin, il peut être noté qu'en fin d'année scolaire, une réunion de tous les partenaires, des responsables pédagogiques et de l'Inspection académique établit une synthèse en complément des premiers bilans sur tablettes.

\section{Poursuite des actions : nouveaux ateliers}

L'offre Nano@school évolue en fonction des programmes scolaires et du développement des nouvelles technologies avec l'objectif de rester très attractif auprès des jeunes. Pour ce faire, l'équipe pédagogique s'attache, chaque année, à faire évoluer les ateliers ou à en proposer de nouveaux. Ainsi les ateliers Microfluidique et Electronique bas coût sont apparus en 2017-2018.

La plateforme «Electronique bas coût » a été créée par les enseignants du centre afin d'une part, de faire comprendre aux lycéens, que derrière la «boite noire » que représente un appareil technologique du quotidien, se cachent des composants ou des systèmes qui réalisent des fonctions unitaires facilement compréhensibles et qui, en général, illustrent avantageusement (car par une approche ludique) des notions scientifiques qui sont à leur programme ; d'autre part de leur permettre de s'approprier ces objets, et même de les détourner pour réaliser leur propre machine, ce qui les rend acteurs de la science et de la technologie.

En 2018-2019, une évolution de l'atelier Nanomonde permet aux lycéens de fabriquer des échantillons de graphène, et de les caractériser grâce à l'utilisation de microscopes à force atomique et à effet tunnel. Cette véritable initiation aux nanosciences, leur permet de comprendre notamment la structure atomique d'un cristal et les forces en jeu à cette échelle. 


\section{Un programme d'envergure qui se dissémine}

Chaque année se sont près de 800 lycéens qui sont sensibilisés au niveau du pôle de Grenoble, ce qui est remarquable quand on sait que cette opération ne rentre pas dans la première mission du pôle microélectronique, structure dédiée prioritairement à la formation dans l'enseignement supérieur.

Cette expérience de sensibilisation est vraiment un modèle unique. Le réseau national CNFM a bénéficié des premières expériences de cette opération Nano@school pour la développer dans d'autres pôles avec des lycéens d'autres académies. Ainsi, les pôles CNFM de Toulouse, Rennes, Paris-Centre, Paris-Sud et Lille se sont lancés dans cette opération de sensibilisation et d'attractivité des jeunes depuis le début du projet IDEFIFINMINA en 2012 (10-12). Chaque année, sur l'ensemble du réseau CNFM, se sont plus de 4.700 lycéens qui sont sensibilisés dont plus de 2.500 avec une expérimentation significative sur plateformes, ce qui constitue un bilan considérable et exemplaire au niveau national. Une vidéo jointe permet de découvrir l'activité des lycéens accueillis dans l'un des pôles du réseau CNFM, dans ce cas le CIME Nanotech. Elle permet de montrer les différents types de plateformes sur lesquelles les lycéens sont sensibilisés et de connaître le point de vue des lycéens et de leurs enseignants :

Presentation_Nano@School_2018-19

Si l'ensemble de la population traitée a toujours manifesté un grand intérêt lors de sa présence, il n'en reste pas moins que l'impact effectif est difficile à mesurer puisque le fruit de ces actions ne pourra se révéler qu'après plusieurs années lorsque ces lycéens seront devenus des étudiants de licences, d'écoles d'ingénieurs ou de masters dans le domaine de l'ingénierie et plus particulièrement de la microélectronique et des nanotechnologies.

Ce sera par un effort collectif de l'ensemble de la communauté éducative quel que soit le niveau, mais aussi grâce à des actions incitatives du côté industriel, ce qui peut être envisagé dans le cadre de la mise en place de la nouvelle Filière Électronique par la Direction Générale des Entreprises rattachée au Ministère de l'Industrie et des Finances, en ce début d'année 2019 (15), que cette expérience pourra se pérenniser et surtout se développer sur l'ensemble du territoire.

\section{Remerciements}

Les auteurs souhaitent remercier le GIP-CNFM (1) et tout particulièrement son pôle Grenoblois, le CIME Nanotech, le CEA-GIANT ainsi que l'Académie de Grenoble pour leur soutien. Ils remercient également tous les enseignants-chercheurs et chercheurs qui sont impliqués dans ce programme, les personnels assurant l'organisation pédagogique et la gestion financière et plus particulièrement Lorraine Chagoya-Garzon qui assume la gestion pédagogique et technique de Nano@School et l'action correspondante du projet IDEFI-FINMINA, ainsi que les stagiaires en alternance successifs. En effet, chaque année un stagiaire en alternance est mis à disposition par le CEA en soutien à la coordination du programme. Les auteurs tiennent à mentionner les soutiens financiers du programme IDEFI-FINMINA, ANR-11-IDFI- 0017, géré par l'Agence Nationale de la Recherche (ANR), mais également d'autres programmes plus régionaux tels que l'IRT Nanoelec et l'IDEX de Grenoble, ce dernier finançant le programme à travers son appel à projet "Summer school". 


\section{Références}

1. O. Bonnaud, L. Fesquet, Communicating and smart objects: multidisciplinary topics for the innovative education in microelectronics and its applications, Oral communication, ITHET 2015 Lisboa (Portugal), 11-13 June, 2015, Proceedings pp. 1-5 DOI: 10.1109/ITHET.2015.7217961

2. O. Bonnaud, New Approach for Sensors and Connecting Objects Involving Microelectronic Multidisciplinarity for a Wide Spectrum of Applications, International Journal of Plasma Environmental Science \& Technology, vol. 10, no. 2, pp. 115-120, 2016, ISSN 2415-0436

3. G. Matheron, Microelectronics evolution, Keynote, European, Microelectronics Summit, Paris (France), Nov. (2014).

4. O. Bonnaud, New Vision in Microelectronics Education: Smart e-Learning and Know-how, a Complementary Approach. (C) Springer International Publishing AG, part of Springer Nature 2019 V. Uskov et al. (Eds.): KES-SEEL-18 2018, SIST 99, pp. 267-275, 2019.

5. F. Papillon, E. Excoffon, E. Martinet, A. Bsiesy, J. Chevrier, Nano@ School : une initiative grenobloise innovante qui place les nanosciences \& nanotechnologies au cœur des interrogations des lycéens et de leurs professeurs, Poster, JPCNFM'2012, Saint-Malo, 28-30 Novembre 2012

6. E. Excoffon, F. Papillon, L. Fesquet, A. Bsiesy, O. Bonnaud, New pedagogical experiment leading to awareness in nanosciences and nanotechnologies for young generations at secondary school, Oral communication; ITHET'2012; Istanbul (Turkey) 21-23 June 2012, Proceedings IEEE ISBN 978-1-4673-2334-5/12, paper n.85, 4 pages

7. O. Bonnaud, T. Mohammed-Brahim, J-M. Floch, A. Bsiesy, Priority of the French national network in microelectronics and nanotechnologies towards the attractiveness of young highschoolers, Oral communication; Proc. of EAEEIE'13, Chania (Greece) 30-31 May 2013, pp.:2227, DOI: 10.1109/EAEEIE.2013.6576495

8. CIME Nanotech : https://cime.grenoble-inp.fr/

9. GIP-CNFM: Groupement d'Intérêt Public - Coordination Nationale pour la formation en Microélectronique et en nanotechnologies. Website: http://wwww.cnfm.fr (Accès mars 2019)

10. IDEFI-FINMINA : Initiative d'Excellence - Formation Innovante en MIcroélectronique et Nanotechnologies, ANR-11-IDFI-0017. Website: http://www.cnfm.fr/VersionFrancaise/actualites/ FINMINA.htm

11. O. Bonnaud, L. Fesquet, P. Nouet, T. Mohammed-Brahim, FINMINA: a French national project to promote Innovation in Higher Education in Microelectronics and Nanotechnologies, Oral communication, ITHET 2014 York (UK), Proc of ITHET'2014; http: / / www.york.ac.uk / conferences / ithet2014 /

12. J.-M. Floc'h, Les actions envers les lycéens dans FINMINA. Présentation orale, JPCNFM'2014, Saint-Malo, 19-21 Novembre 2014

13. O. Bonnaud, Bsiesy, E. Martinet, R. Baptist, S. Basrour, E. Pernot, Increasing Attractiveness of Electrical Engineering for Schoolers Through Experiments on Microelectronics and Nanotechnology Interuniversity Platforms. Oral presentation, Proc. 28th EAEEIE Annual Conference (EAEEIE), 26-28 Sept. 2018, Hafnarfjordur, Iceland IEEExplore, 10.1109/EAEEIE.2018.8534281, 4 pages 2018

14. O. Bonnaud, A. Bsiesy, L. Fesquet, B. Pradarelli, IDEFI-FINMINA: a French educative project for the awareness, innovation and multidisciplinarity in microelectronics, Proc. of EAEEIE'2017 conference, 7-9 June 2017, Grenoble (France)

15. DGE et Filière Electronique : https://www.entreprises.gouv.fr/conseil-national-industrie/la-filiereindustries-electroniques

\section{Note à l'éditeur}

Vidéo : animation vidéo-sonore, format avi :

Titre : Presentation_Nano@School_2018-19.avi 